\title{
GENERATION OF MICRO-SIZED CONDUCTIVE LINES ON GLASS FIBRE FABRICS BY INKJET PRINTING
}

\author{
Unai Balda Irurzuna, Victoria Dutschk ${ }^{\mathrm{a}^{*}}$, Alfredo Calvimontes ${ }^{\mathrm{b}}$, Remko Akkerman $^{\mathrm{a}}$ \\ aFaculty of Engineering Technology, University of Twente, Postbus 217, 7500 AE Enschede, the Netherlands \\ ${ }^{*}$ E-mail address: v.dutschk@utwente.nl \\ bLeibniz-Institut für Polymerforschung Dresden e.V., Hohe Strasse 6, 01069 Dresden, Germany
}

\begin{abstract}
:
Micro-sized lines were inkjet printed on glass fibre fabrics using different droplet spacing. A conductive ink containing silver nanoparticles was used in this study. Glass fibre fabrics were differently pre-treated to avoid spontaneous spreading of the ink dispersion. The sample topography was examined using scanning electron, optical and confocal microscopy with a chromatic sensor. Printability conditions were discussed based on the results of topographic characterization and wettability measurements.
\end{abstract}

\section{Key words:}

Glass fibre fabric, inkjet printing, conductive lines.

\section{Introduction}

Electronic fabrics, also called e-textiles, is an emerging topic in the field of smart textiles [1,2]. Electronics can be integrated into textiles in different ways, i.e. integrating micro-systems with connectable modules in clothes or textiles as well as integrating electronic functions into the fibres or onto the fabric surface. Inkjet printing is one of the most promising techniques by which the circuit components can ultimately be drawn onto the substrate in one step [3-5].

In the present study, the inkjet printing technique was used to deposit silver nanoparticles in order to obtain micro-sized conductive lines on glass fibre fabrics. It is well known that ink viscosity and surface tension are crucial parameters in the preparation of an ink formulation [6]. On the one hand, the ink viscosity has to be low enough to allow the channel to be refilled in about $100 \mu \mathrm{s}$, whereas the surface tension has to be high enough to hold the ink in the nozzle without dripping. On the other hand, the wettability of a substrate to be inkjet printed has to be appropriate to avoid ink spreading over the substrate while printing. Having in mind that glass fibre surface is rather hydrophilic with a pronounced polar character $[7,8]$, the glass fibre fabrics were differently hydrophobized.

Scanning electron microscopy was used to obtain qualitative information about samples' surface topography and morphology. A non-contact optical technique was additionally applied for topographic characterisation of the samples. To obtain the hydrophobicity degree of glass fibre fabric surfaces before and after treatment, dynamic wetting measurements were performed. The printability of conductive lines onto glass fibre fabrics was discussed based on the results of topographic characterization and wettability measurements.

\section{Materials and methods}

In the present study, a tetradecane dispersion of silver nanoparticles was used as an ink (Harima, Japan). The nanoparticles diameter is about $5 \mathrm{~nm}$, and their amount is about $60 \%$ of the ink weight. A glass fabric SS 303 (TenCate, Netherlands) made from E-glass fibres was used as substrate with a weight of $300 \mathrm{~g} / \mathrm{m}^{2}$, a filament diameter of $6 \mu \mathrm{m}$ and the weight ratio between warp and weft of 51:49.

\section{Substrate preparation}

Glass fibre fabric surfaces were modified in two ways: (i) with hexamethyl disilizane (HMDS) and (ii) with amorphous Teflon AF. In the first case, the glass fibre fabric was hydrophobized for 2 hrs in HDMS vapours. In the second case, the samples preliminarily treated with HDMS were modified with Teflon AF by dip coating. A 6\% Teflon AF $1601 \mathrm{~S}$ solution (Du Pont) in an FC-75 solvent (3M) was used after it was diluted fivefold with the same solvent. Finally, the Teflon AF coating was dried for $12 \mathrm{~h}$ in vacuum at $25^{\circ} \mathrm{C}$ and then for $1 \mathrm{~h}$ at $165^{\circ} \mathrm{C}$ as recommended by the producer until the solvent was removed completely. This procedure and chemical details of Teflon AF are described in more detail elsewhere [9].

The inkjet printing of colloidal silver particles was performed with an inkjet printer Jetlab 4 (MicroFab Technologies Inc., USA). The droplet pitch was reduced in order to facilitate the contact between the droplets. As the dimensions of the substrate are not subjected to any standard, the starting point of the pattern was different for the substrates studied. The direction of the weft yarn was taken into account. The temperature of the substrate holder was set to $70^{\circ} \mathrm{C}$ in order to minimize ink spreading over the substrate. To obtain a continuous structure, the deposited material was sintered at $210^{\circ} \mathrm{C}$ for 60 minutes as specified by the ink supplier. This process is extremely important, as it will define the microstructure of the printed pattern. The more continuous the microstructure is, the better the conductivity of the final product. Cleaning the nozzle after using the silver-based ink requires a suitable solvent. In this study, hexane was used as a cleaning solvent. After printing, the sample was introduced in a convection oven in order to cure it.

\section{Surface characterization}

Sample topography and morphology was studied using a Neoscope JCM5000 scanning electron microscope (JEOL, Japan) as well as with a Leica M125 optical stereo microscope (Leica, Germany). 
Sample surface topography was examined using a MicroGlider imaging measuring instrument for the optical roughness analysis (FRT, Germany), operating on the principle of chromatic aberration. This measuring method was described in more detail elsewhere [10].

To obtain the degree of hydrophobicity of glass fibre fabrics before and after modification, dynamic wetting measurements were carried out with a FibroDAT 1122HS dynamic contact angle tester (Fibro System, Sweden). Some advantages of this equipment over other contact angle measuring systems as well as the measuring procedure were detailed previously [11]. The instrument was located in a temperature-controlled laboratory maintained at $23 \pm 1^{\circ} \mathrm{C}$. Relative humidity of $40 \pm 2 \%$ was kept constant.

\section{Results and discussion}

First, conductive lines were printed without pre-treating the sample. Figure 1 represents a conductive line printed once in the warp yarn direction. As is clearly seen, the line printed is not continuous and cannot serve as a conductive track. The distance between droplets is too large if printed in the warp direction; no contact between droplets was achieved. However, after printing silver nanoparticles several times at the same position, the layer which should be conductive becomes visible. The light grey colour indicates that the conductive layer is building up. Figure 2 shows a line printed several times in the weft direction. The distance between droplets illustrated in Figures 1 and 2 is the same. Printing in the weft direction guarantees the continuity of the track, i.e. the droplets are connected with each other, while printing several layers in the warp does not guarantee continuity. The continuity of the track starts to build up when the gaps between the fibres are filled in with the ink. The need for at least three layers was revealed by measuring conductivity signals.

a)

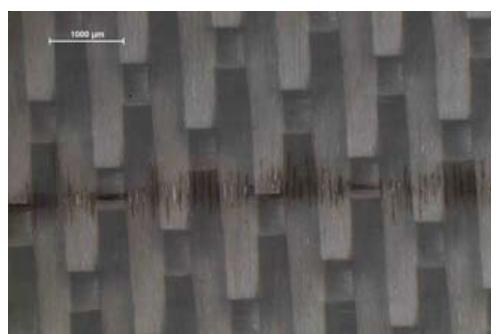

b)

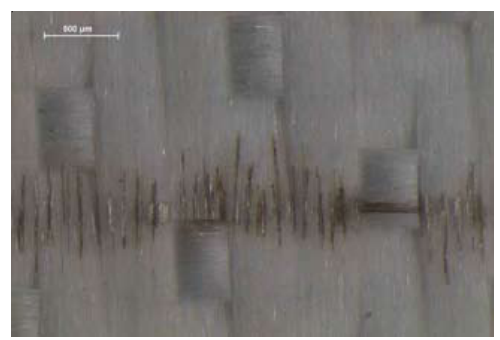

C)

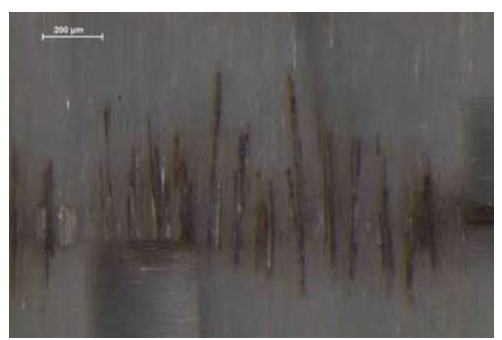

Figure 1. Optical microscope images of a glass fibre fabric with a line containing silver nanoparticles printed in the warp direction: (a) magnification 20X; (b) magnification 40X and (c) magnification $80 \mathrm{X}$. a)

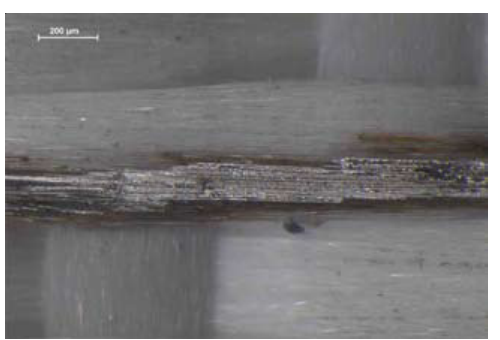

b)

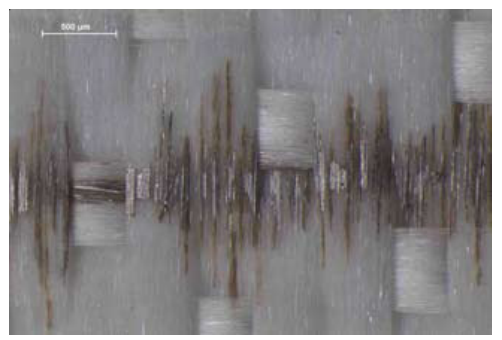

C)



Figure 2. Optical microscope images of a glass fibre fabric with an inkjet-printed line, which was printed several times at the same position in the weft direction: (a) printed 6 times; magnification 80X; (b) printed 3 times; magnification 40X and (c) printed 3 times; magnification $80 \mathrm{X}$.
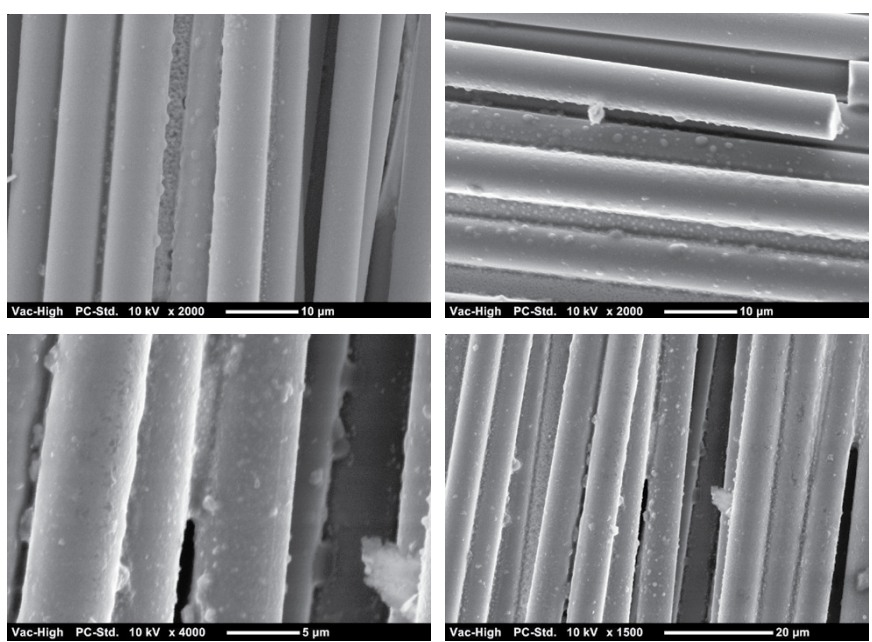

Figure 3. SEM images of the printed structure on an untreated glass fibre fabric at different positions and different modifications.

Figure 3 shows the distribution of the ink over the fibres. The structure was printed once. The gaps are filled in with the ink and the continuity of the line starts to build up within the gaps. When the gaps are fully filled, the line continuity starts to build up at the surface. In order to decrease the ink distribution among the fibres, the temperature to heat the substrate while printing was increased to $80^{\circ} \mathrm{C}$. The results were insignificant compared to those printed without heating the substrate. For applications where the profile shape is important, such as radio-frequency identification (RFID) tags or strain gauges, the results achieved are not acceptable. Moreover, the use of a multi-meter confirmed that conductivity is only guaranteed between the warp yarns below the weft yarn, and only in those 
cases where printing was carried out in the weft yarn direction. The results revealed that surface pre-treatment was needed to print lines with the conductivity function.

To achieve a better adhesion between the Teflon AF coating and the glass fibre fabrics, a pre/treatment with HMDS is needed, as described previously in [9].

SEM images of the treated glass fabric (Figure 4) show that the coating layer was built on the surface. It is noticeable that the gap between two consecutive fibres does not exist any more. Different types of lines were printed, starting from just a single layer until printing three times on top of ten consecutive lines next to each other. The figures below are pictures taken with a stereo microscope.
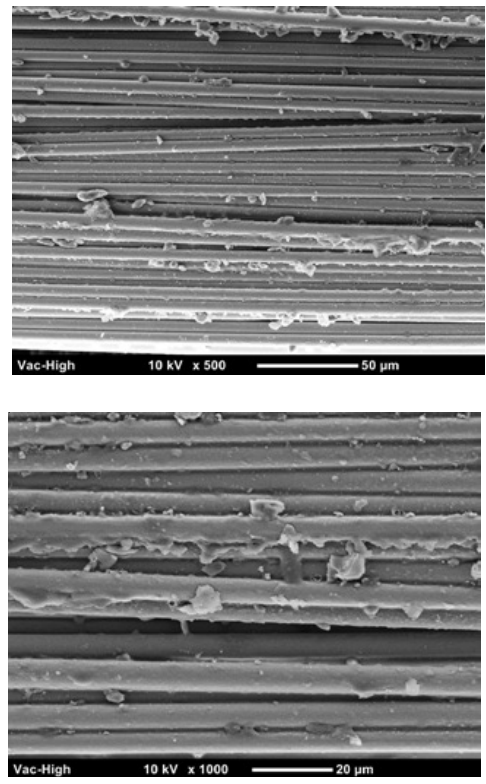

Figure 4. SEM images of the treated glass fabric taken at different magnifications.

a)

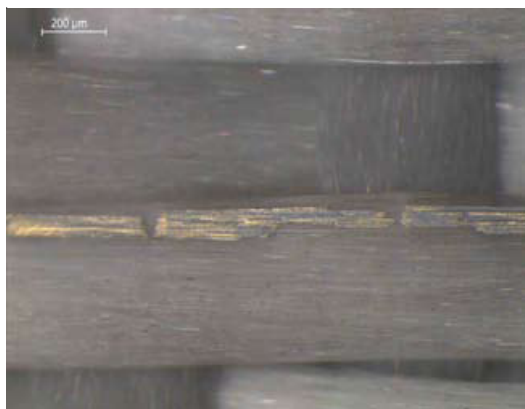

b)

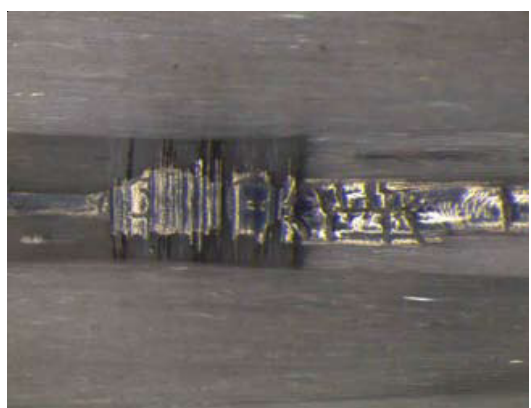

Figure 5. Microscopic images of inkjet-printed conductive lines: (a) one-layer conductive track; (b) 5 layers printed consecutively on each other.
Figure 5a represents a one-layer conductive track. It can be clearly seen that the ink does not penetrate between the fibres, but remains on the surface. Figure $5 b$ shows 5 layers printed consecutively on each other. As can be seen, the warp yarn is too high and the printed line cannot overtake it. Here, the continuity was lost.

However, if 10 consecutive lines were printed next to each other, the results show that the continuity is guaranteed from one end of the line to the other (Fig. 6). The assumption is proved using a multi-meter, by measuring resistance values of $1-25 \Omega$ depending on the number of layers printed.

a)

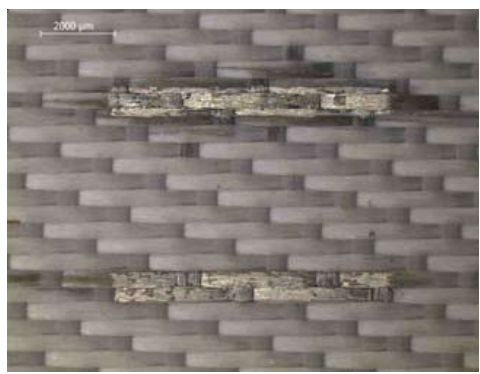

b)

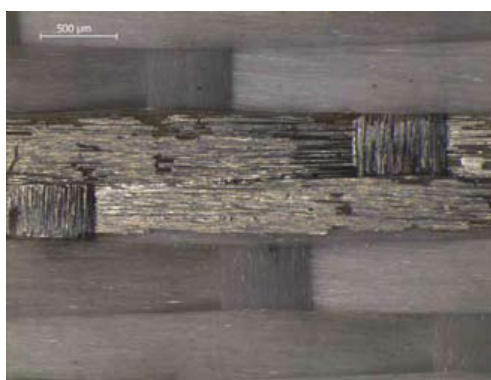

Figure 6. Microscopic images of 10 times inkjet-printed conductive line taken at different magnifications.

a)

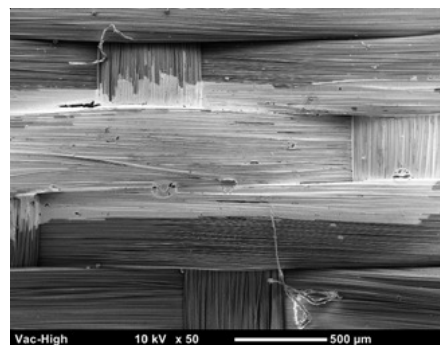

b)

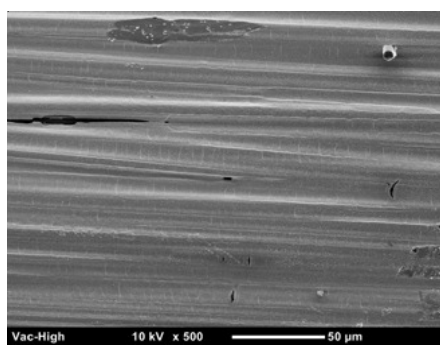

c)

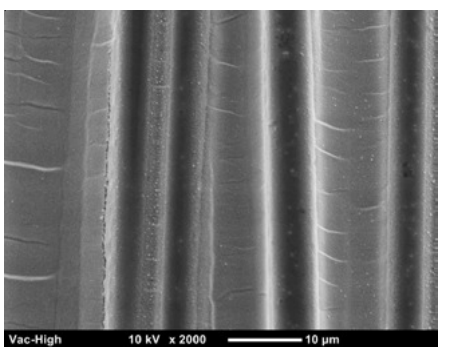

Figure 7. SEM images of 10 times inkjet-printed conductive line taken at different magnifications. 
SEM images are in agreement with the conclusion: the fibres are fully covered by the ink (Fig. 7). The places not covered are fully visible in black colour and they look as if they have cracks.

The shape accuracy of the printed pattern is quite acceptable. As can be seen in Figure 6, the rectangular profile of the printed structure is noticeable. Nevertheless, the printed structure exceeds the height of the warp yarn as can be seen in Fig. 7c. This is due to the large amount of the ink.

Images performed using optical and scanning electron microscopy reinforced the hypothesis that a coating film is formed on the glass fibre fabric after being hydrophobized with HMDS and Teflon AF. The following images were obtained using a MicroGlider chromatic confocal microscope (Bergisch Gladbach, Germany) equipped with a chromatic sensor described elsewhere [10].

a)
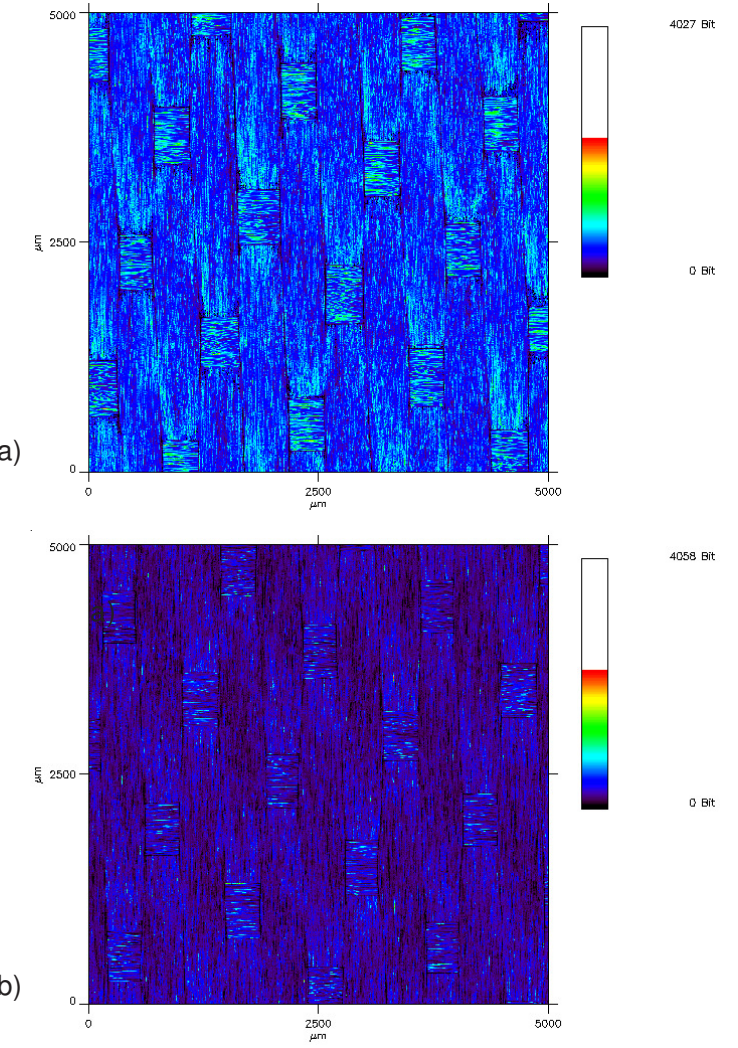

Figure 8. Images taken with a MicroGlider confocal microscope showing the intensity map for: (a) unmodified glass fibre fabric and (b) modified with HMDS and Teflon AF.

Figure $8 \mathrm{a}$ shows the intensity map of an unmodified glass fabric substrate. The differences in colour are the mean differences in light reflection. Therefore, the intensity map is indirectly related with the topography of the substrate. On the other hand, Figure $8 \mathrm{~b}$ shows the intensity map of the same substrate modified with HMDS and Teflon AF. The differences in light intensity are notable. These changes in colour intensity indicate changes in the chemical structure, since Teflon AF and HMDS are absorbing light. Increasing the resolution of the microscope, the topography of the filaments was measured. For that purpose, a High Resolution ScanDisk Confocal Microscope (SDCM) was used. Figure 9a represents the profile of an unmodified and modified warp filament. As illustrated, the surface roughness was increased due to surface modification.

The same effect is described in the weft filament (Fig. 9b). The roughness profile undulation on the weft yarn is due to the
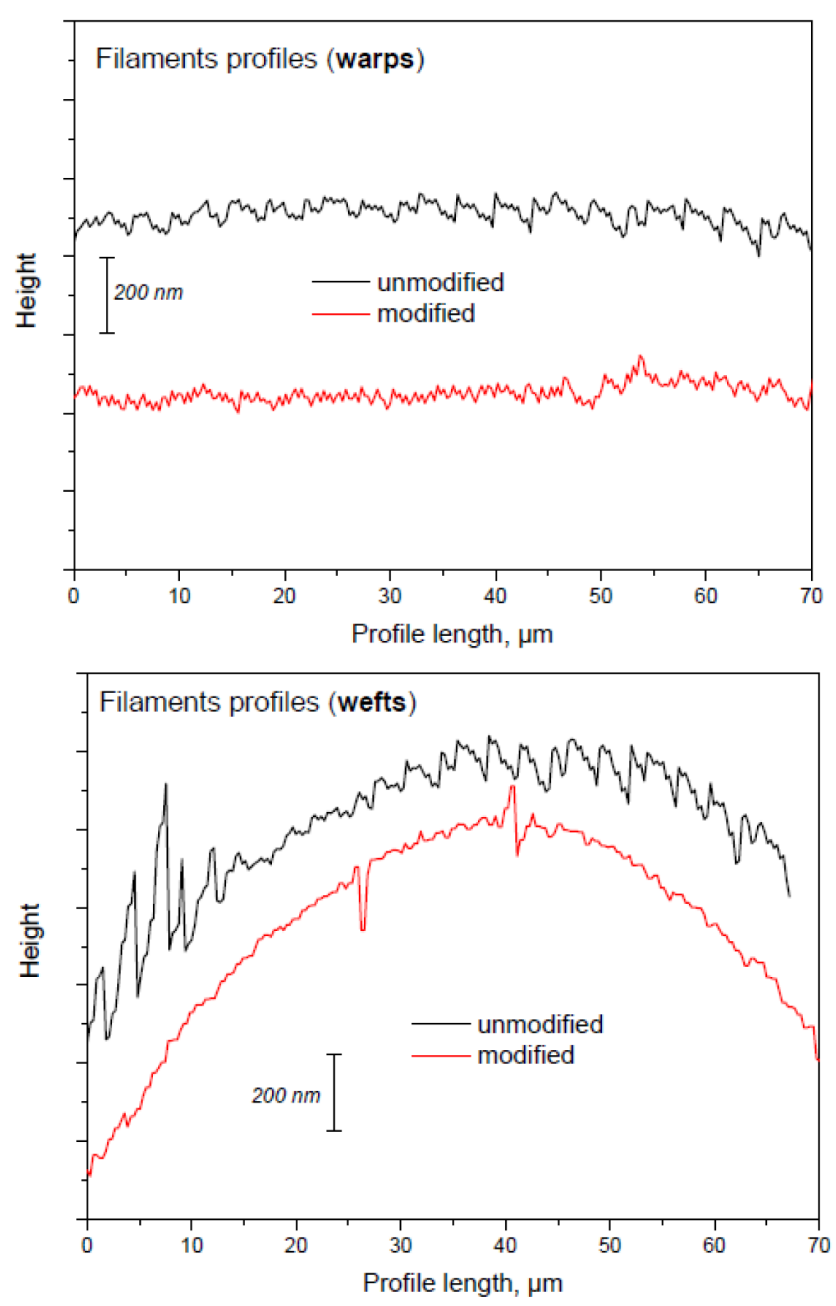

Figure 9. Profiles calculated from the images taken using the confocal microscopy: warp (up) and weft (down).

a)

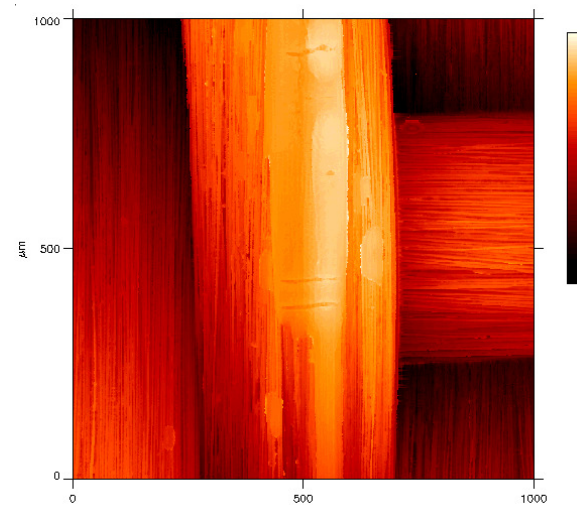

b)

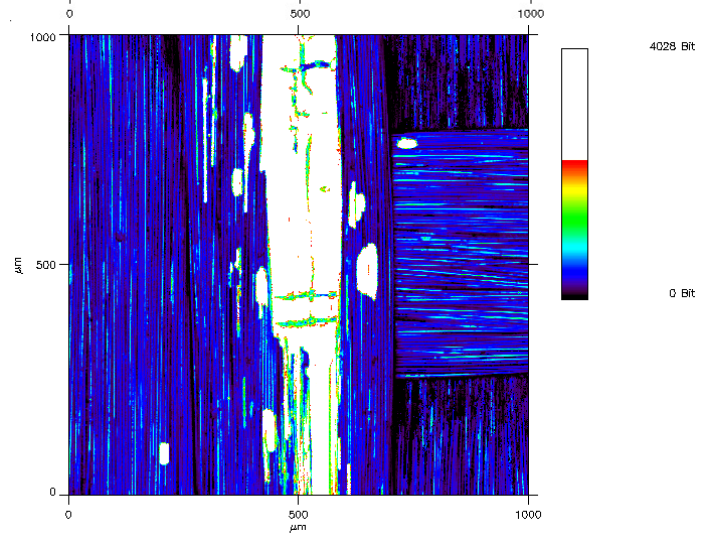

Figure 10. Images of the printed structure using a MicroGlider chromatic confocal microscope (a) information on topography; (b) information on light intensity 


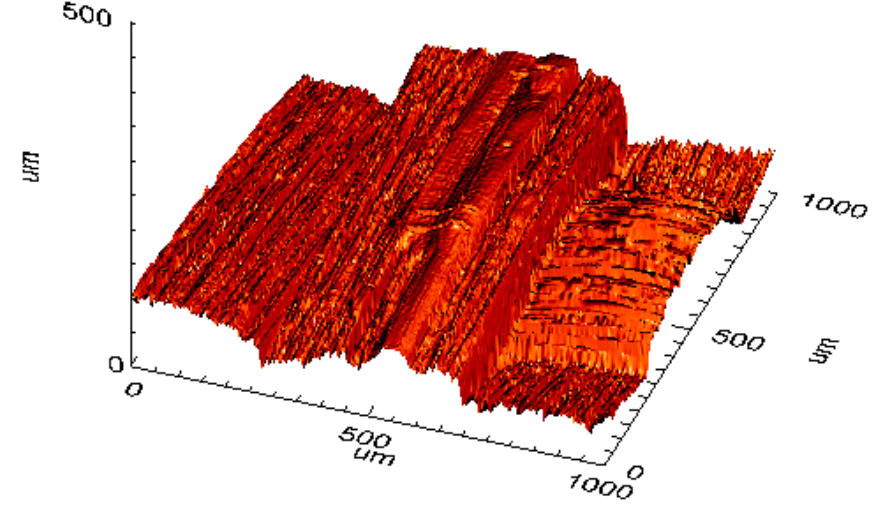

Figure 11. 3D image of the structure printed taken by MicroGlider.

shape that the weft yarn forms. At the same time, the chromatic white light $(C W L)$ sensor allows a conductive layer of the printed structure to be visualised by measuring the light reflection. Figure 10a illustrates the printed structure. Changing the intensity of the lamp's light permits a better view of the printed conductive layer (Fig. 10b). The printed structure can be seen in Fig. 11 as a 3D image.

In order to measure the thickness of the printed structure, the Chromatic White Light (CWL) confocal microscope was used. Although this microscope is mostly used to measure roughness, it permits the measurement of height differences. The pictures on the right characterize the height difference of the printed structure compared with the surface of the glass fabric. While Figure 12a illustrates where the measurement was done, Figure $12 \mathrm{~b}$ is a representation of the measured height difference.

a)

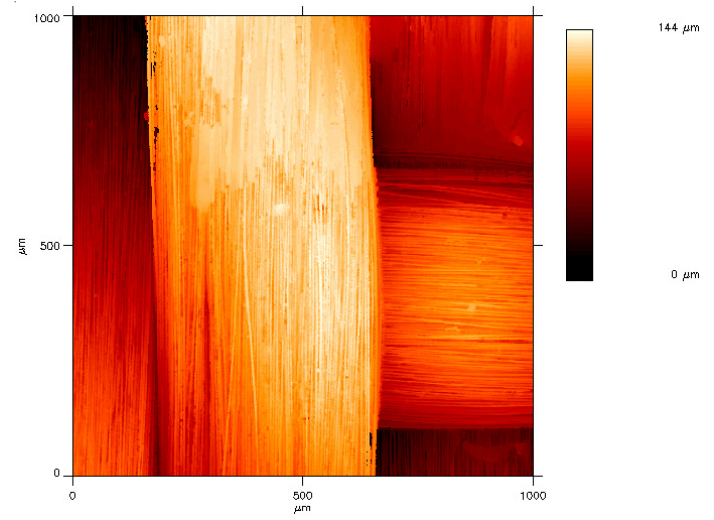

b)

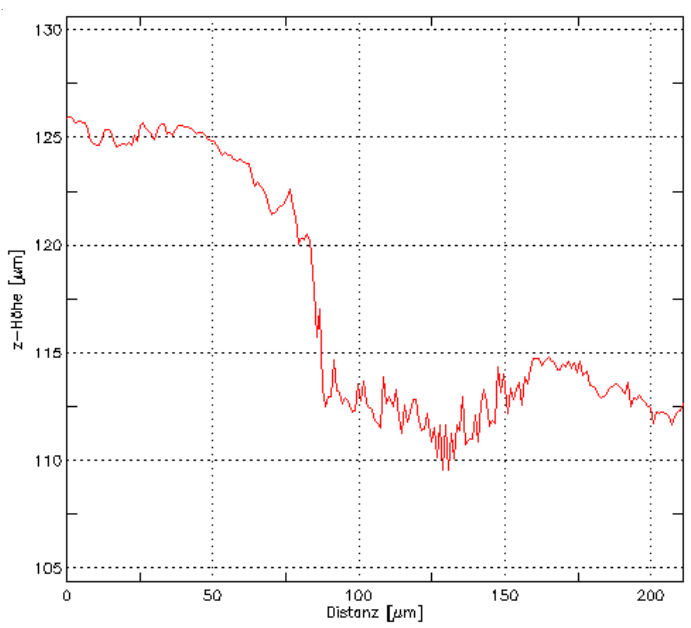

Figure 12. Images of the printed structure using a MicroGlider chromatic confocal microscope (a) information on topography; (b) profile analysis.
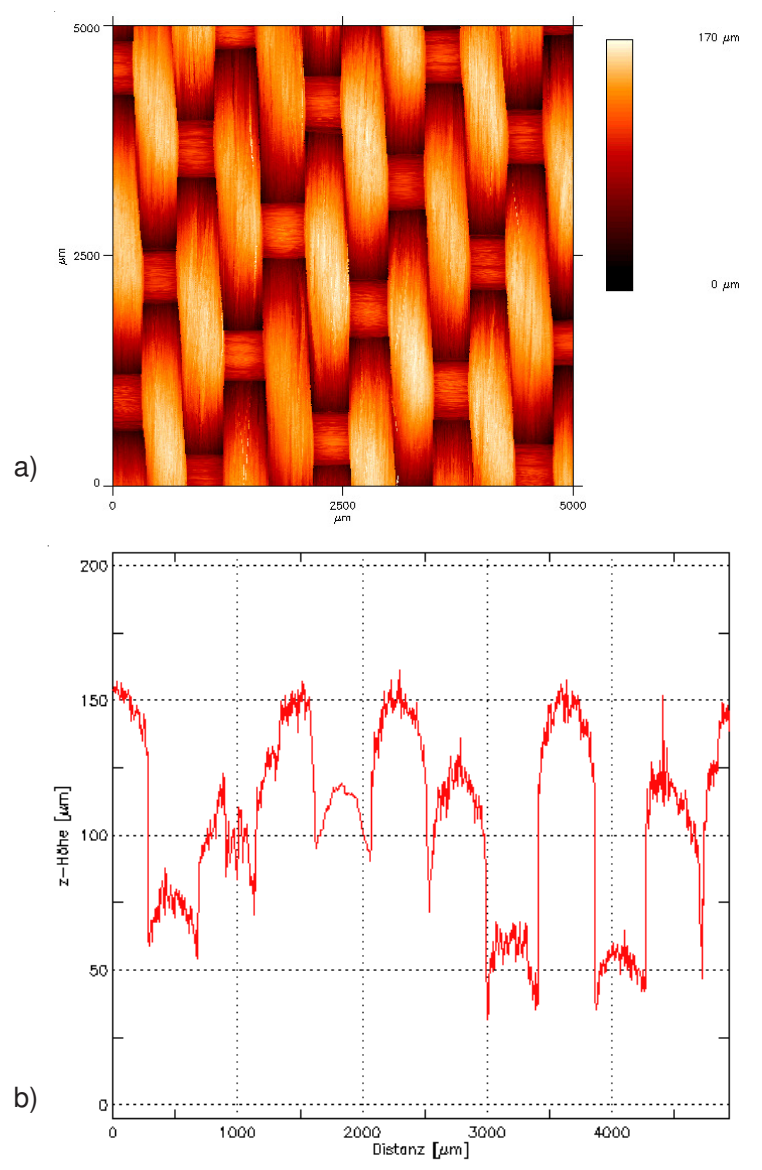

Figure 13. Images of the printed structure using a MicroGlider chromatic confocal microscope (a) information on topography; (b) profile analysis.

The result is a measured thickness of about $10 \mu \mathrm{m}$. This is a considerable layer thickness compared with structures printed on flat surfaces. The reason is the number of layers printed, in this case 5 , and on the other hand, the surface modification of the fabric increasing the contact angle of the droplets noticeably.

The height differences visualized during printing sessions that avoid the creation of single droplet width conductive structures, indicate the need to measure the height difference between weft and warp yarns in order to quantify at which point the printability of conductive tracks on fabrics becomes critical.

As explained before, the CWL microscope offers the opportunity to characterize the fabrics' topography. Figure 13 represents the surface of the glass fabric under study. The black line indicates in Fig. 13a the place where the profile of the surface was measured. In Fig. 13b, the corresponding surface roughness profile is shown.

Furthermore, a height difference of $50 \mu \mathrm{m}$ was measured between the weft yarn and warp yarn related with the printing on the weft yarn direction (Figures 14a and 14b). They are interesting values which provide a guideline of which height difference cannot be exceeded by a single printed line. From this measurement, it was concluded that the height difference between weft and warp yarns is critical in order to achieve proper conductive tracks. The smaller the height difference, the easier it will be to exceed the difference and create a conductive layer for the printed structure.

In order to measure the wettability of the modified and unmodified glass fabric, a FibroDAT 1122 dynamic contact 
a)

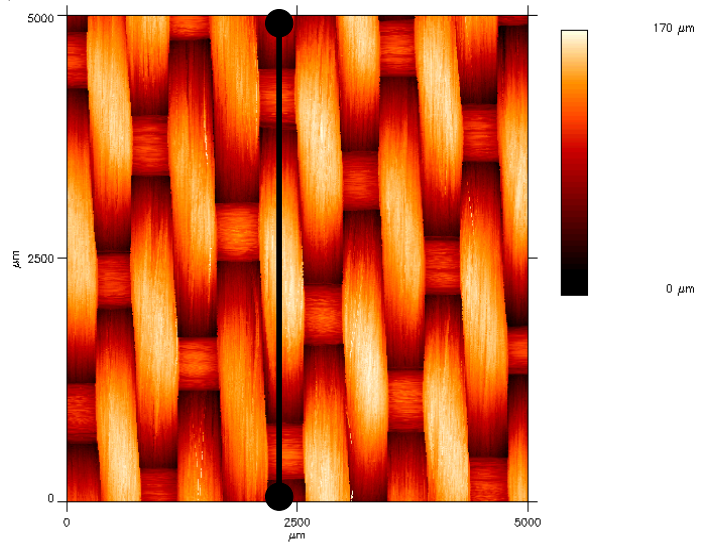

b)

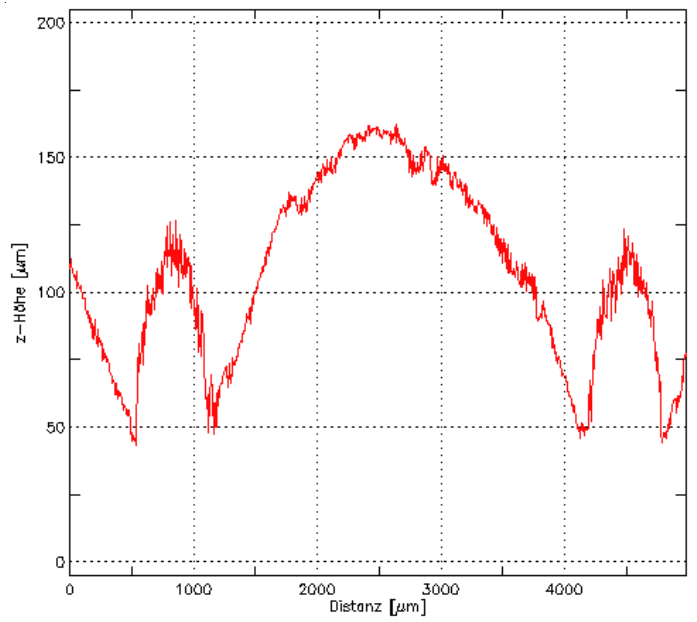

Figure 14. Images of the printed structure using a MicroGlider confocal microscope (a) information on topography; (b) profile analysis.

angle tester (Fibro System, Sweden) was used. Dynamic wetting measurements were carried out in order to estimate the degree of hydrophobicity. On the basis of macroscopic water drop base changes, the wetting behaviour of the water drop can be divided into three different regimes: (a) dynamic wetting (spreading); (b) quasi-static wetting and (c) penetration.

Figure 15 shows the three different regimes of the wetting behaviour on modified glass fibre fabrics. Figure 15 represents the dynamic wetting behaviour of a water droplet on modified and unmodified glass fabrics. On the unmodified glass fabric, the three regimes are clearly visible, while on the modified glass fabric, penetration was avoided due to the hydrophobic coating created by modification with Teflon AF and HMDS. Figure 15b illustrates the water contact angle as a function of time. The oscillations measured at the modified substrate are due to the potential energy stored on the droplet when it falls from the nozzle. Within three seconds, a constant contact angle of around $117^{\circ}$ was measured.

\section{Conclusions}

In the present study, the printability of a conductive ink containing silver nanoparticles on glass fibre fabrics was studied. It was shown that the ink used spreads over rather hydrophilic glass fibre fabric, not allowing a continuous conductive line to be printed. There are two possible ways to solve the problem: (i) to modify the ink, changing its surface tension and viscosity and (ii) to modify the substrate to be printed. In this study, the latter was chosen. The glass fibre fabric was hydrophobized in two different ways. Using dynamic wetting measurements, the changes in chemical composition
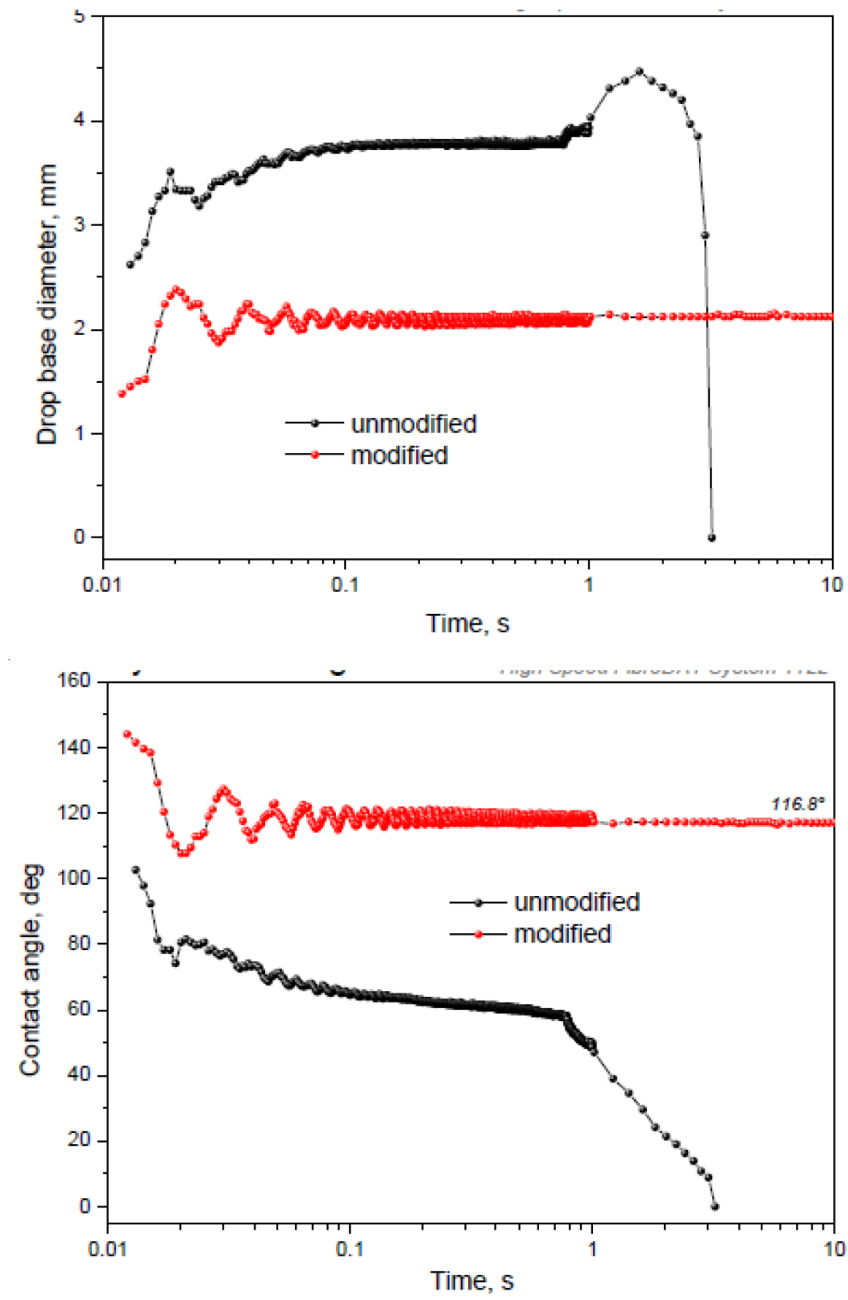

Figure 15. Dynamic behaviour of a droplet on modified and modified glass fibre fabric: (up) drop base diameter as a function of time; (down) contact angle a s a function of time.

of the substrate before and after modification were indirectly shown, using the contact angle concept. The use of different microscopic methods to evaluate the surface topography with and without conductive track printed allowed a conclusion to be drawn about a relationship between the properties of functional inks and the textile properties of fabrics used.

\section{Acknowledgement}

This research was supported by the ESA project FASES 'Fundamental and Applied Studies in Emulsion Stability' AO99-052.

\section{References:}

1. Locher, I., Trosler, G., Textile Research Journal 77 (2008) 837.

2. van Langenhove, L., Hertleer, C., Catrysse, M., Puers, R., van Egmond, H., Mattijs, D., in: Wearable eHealth Systems for Personalised Health Management (Eds. A. Lymberis, D. de Rossi), IOS Press 2004, p. 344-352.

3. Zhang Chunming, Fang Kuanjun, Surface \& Coating Technology 203 (2009) 2058.

4. Bidoki, S. M., Nouri, J., Heidari, A. A., Journal of Micromechanics and Microengineering 20 (2010) DOI: 10.1088/0960-1317/20/5/055023. 